\title{
Design and Simulation Analysis of an Energy-Saving Type of Overrunning Clutch Life Testing Device
}

\author{
Lihua Zhong, Shiyou Zhang, Chao Xie and Jin Yao* \\ School of Manufacturing Science and Engineering, Sichuan University, Chengdu 610065, China \\ ${ }^{*}$ Corresponding author
}

\begin{abstract}
A new type of energy-saving life testing device used for overrunning clutch, with simple structure, symmetrical layout and low energy consumption, is proposed to simplify the testing system and solve the problems of complexity, bulkiness and high cost, which exist in the traditional comprehensive testing systems. The interaction between two groups of the inverse cam mechanism are utilized to automatically achieve the state transition of overrunning clutch, the torsion springs to load overrunning clutch, and a flywheel to reduce speed fluctuations. Therefore the complex control system and data acquisition system are not required. The functions of the device are illustrated and verified with a 3D model of the device and its kinematics and dynamic simulation.
\end{abstract}

Keywords—overrunning clutch; life testing; simulation analysis; energy-saving

\section{INTRODUCTION}

Overrunning clutch is an important component for dynamic transmission, which is widely used in many mechanical transmission systems. It is a device that engages and disengages automatically according to the relative velocity between the driveshaft and the driven shaft. The present overrunning clutch testing systems are composed of drive system, clutch installation system, load system, control system and data acquisition system. The working process of the testing systems is as follows: a) The drive system provides energy to the overrunning clutch. b) The control system controls the movement of the testing system according to the data which collected from every parts. c)The load system provides the required load when the clutch is engaged. Zhu[1] and Tie[2] used hydraulic system to load in their overrunning clutch testing devices. These testing devices have the advantages of big loading force and small impact force. However, the whole testing device is bulky, has a slow response speed, requires extra load and has a large energy consumption. Burgess[3] and Imado[4] used brake to load in their clutch testing devices. In these testing device, the load is adjustable but it costs much energy. Richter[5], Li[6] and Liu[7] designed testing systems that use two motors to simulate the operating conditions of the overrunning clutch. These testing systems have the characteristic of high rotation speed and can be controlled automatically by program. However, these testing systems require complex control system and the cost is high. Cramton[8] designed a testing device using non-circular gears and a torsion spring to load back and forth between two overrunning clutches every halfrevolution. The device can test two clutches at a time, but the energy of the device is finally consumed by the internal friction of the multi-disc clutch. In general, the above clutch testing devices have the problem of high energy consumption or high cost or complex control system.

In this paper, a new type of energy-saving life testing device for overrunning clutch, with simple structure, symmetrical layout and low energy consumption, is proposed. The system uses the combination of the inverse cam mechanism and torsion springs to achieve the state transition and the load of overrunning clutch. The design and analysis results of the device show that this device is able to achieve the function of the overrunning clutch life testing systems and save energy, which provides the basis for the design of the same type of test device.

\section{FUnCtional REQUiREMENTS OF THE TESTING DEVICE}

The structure of the overrunning clutch is shown in Figure I. During the working process, there will be an engaged state and a disengaged state. When the inner race rotates counterclockwise relative to the outer race, the clutch is in the engaged state where the inner race and the outer race are in the same speed, and there is a load between the inner race and the outer race. When the inner race rotates clockwise relative to the outer race, the clutch is in the disengaged state, and there is no load between the inner race and the outer race.

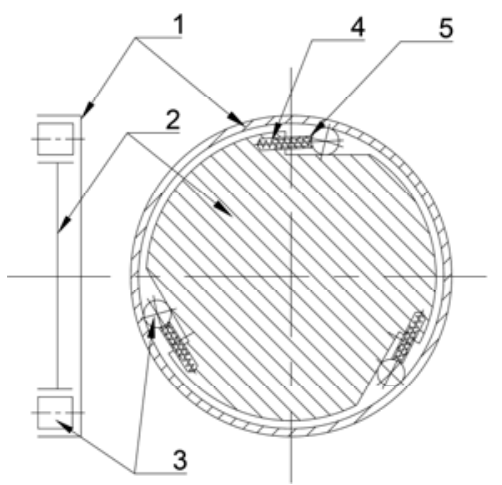

FIGURE I. STRUCTURE OF THE OVERRUNNING CLUTCH. 1 OUTER RACE, 2 INNER RACE, 3 ROLLER, 4 SPRING, 5 PLUNGER.

In this paper, when designing the overrunning clutch life testing device, not only the transition of the two states but the way to improve testing efficiency and reduce the energy consumption are considered. The specific requirements are as follows: 
It must be able to achieve the transition of the two working states.

When the clutch is in the engaged state, there will be a load and the load should be adjustable so that the testing system can test various of clutch.

In order to control the testing time, the transition frequency of the two working states should be adjustable.

It should be a low energy consumption device which means using the minimum energy to complete the testing function.

Its structure is simple and it does not need complex control systems.

\section{Structure AND PRinciple of the Testing DeVICE}

\section{A. Structure of the Testing Device}

The structure of the proposed energy-saving overrunning clutch life testing device is as shown in Figure II. The device consists of a flywheel, two groups of inverse cam mechanism, two overrunning clutches, two torsion springs and a group of gear. The inverse cam mechanism $\mathrm{A}$ is composed of cam A, follower $A$ and rollers, and the inverse cam mechanism $B$ is composed of cam B, follower B and rollers. The flywheel with a certain counterclockwise rotation speed is fixed to shaft A, and it provides energy for the testing device(the flywheel is driven by a motor which is not drawn in Figure II). Shaft B's rotation speed and direction is the same as shaft $\mathrm{A}$, which are ensured by the gear group connecting shaft $\mathrm{A}$ and $\mathrm{B}$. The rotation speed and direction of follower $A$, follower $B$, outer race of clutch $A$, outer race of clutch $B$, which are fixed on the shaft A and B respectively, are the same as the flywheel during the entire testing period.

\section{B. Principle of the Testing Device}

As is shown in Figure II(b), follower A drives cam A clockwise through a roller, which makes the non-preload torsion spring A rotate clockwise to store energy and the inner race of clutch B rotate clockwise. At this time, follower B is just separated from cam $B$ which has the trend of making inner race of the clutch $A$ rotates counterclockwise with the effect of the maximum preload torsion spring $B$. Then clutch $B$ is in the disengaged state and clutch $\mathrm{A}$ is in the engaged state accordingly. The stiffness coefficient and preload of the torsion spring determine the load of the overrunning clutch during the testing process. Inversely, when the flywheel rotates counterclockwise 90 degrees, follower $A$ is just separated from cam A, and the clutch B is transferred from the disengaged state to the engaged state. Follower $\mathrm{B}$ drives cam $\mathrm{B}$ with clockwise rotation through a roller, and the clutch $\mathrm{A}$ is transferred from the engaged state to the disengaged state. The torque of torsion spring $\mathrm{A}$ is reduced to zero, and the toque of torsion spring $B$ increases to the maximum. The testing device will cycle through 90 degrees.

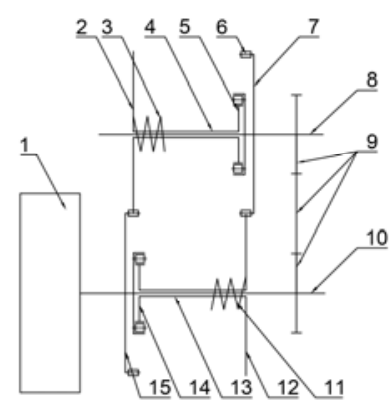

(a)

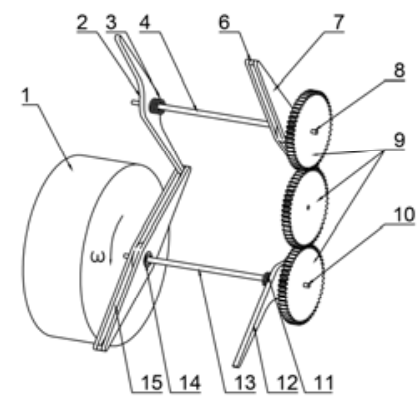

(b)
FIGURE II. STRUCTURE OF THE DEVICE. (A) 2D MODEL, (B) 3DMODEL. 1 FLYWHEEL, 2 CAM A, 3 TORSION SPRING A, 4 HOLLOW SHAFT A, 5 OVERRUNNING CLUTCH A, 6 ROLLER $(\times 4), 7$ FOLLOWER B, 8 SHAFT B, 9 GEAR GROUP, 10 SHAFT A, 11 TORSION SPRING B, 12 CAM B, 13 HOLLOW SHAFT B, 14 OVERRUNNING CLUTCH B, 15 FOLLOWER A.

\section{SimUlATION OF KinEMATICS AND DYNAMICS OF THE Testing DeVICE}

To verify the proposed life testing device, modeling, simulation and analysis are carried out in ADAMS (Automatic Dynamic Analysis of Mechanical Systems) software.

\section{A. The Basic Experiment Parameters of the Overrunning Clutch}

According to the Mechanical Design Handbook[9], the CKA3 overrunning clutch is selected and its basic parameters are shown in Table I.

TABLE I. BASIC PARAMETERS OF THE CKA3 OVERRUNNING CLUTCH

\begin{tabular}{|c|c|}
\hline Torque $(\mathrm{N} \cdot \mathrm{m})$ & 63 \\
\hline The maximum speed(r/min) & 2000 \\
\hline Outer race diameter $(\mathrm{mm})$ & 60 \\
\hline Inner race diameter(mm) & 20 \\
\hline Width $(\mathrm{mm})$ & 22 \\
\hline
\end{tabular}

B. Modeling and Testing Parameters of the Testing Device

The 3D model of the device is build by Solidworks software. Then the model is imported into the ADAMS software. After simplifying the overrunning clutch, adding constraint and friction moment of the testing device, the kinematics and dynamics simulation are carried out. The main testing parameters of the simulation model are shown in Table II.

TABLE II. THE MAIN TESTING PARAMETERS OF THE SIMULATION MODEL

\begin{tabular}{|c|c|}
\hline Part & Initial parameter value \\
\hline $\begin{array}{c}\text { Torsion } \\
\text { spring A }\end{array}$ & Stiffness coefficient: $700 \mathrm{~N} \cdot \mathrm{mm} / \mathrm{deg}$, preload:63 $\times 10^{4} \mathrm{~N} \cdot \mathrm{mm}$ \\
\hline $\begin{array}{c}\text { Torsion } \\
\text { spring B }\end{array}$ & Stiffness coefficient: $700 \mathrm{~N} \cdot \mathrm{mm} / \mathrm{deg}$, no preload \\
\hline Flywheel & $\begin{array}{c}\text { The moment of inertia: } \mathrm{J}_{1}=4.596 \times 10^{7} \mathrm{~kg} \cdot \mathrm{mm}^{2} \text {, initial } \\
\text { angular velocity: } 200 \mathrm{deg} / \mathrm{s}\end{array}$ \\
$\begin{array}{c}\text { The moment of inertia: } \mathrm{J}_{2}=3.675 \times 10^{7} \mathrm{~kg} \cdot \mathrm{mm}^{2}, \text { initial } \\
\text { angular velocity: } 200 \mathrm{deg} / \mathrm{s}\end{array}$ \\
\hline
\end{tabular}




\section{1) Simplification of the overrunning clutch}

In the simulation model, the overrunning clutch is simplified as an inner race and an outer race, and the constraint between the inner race and the outer race is controlled by setting two sensors and a script simulation program. One sensor measures the relative speed between the inner race and the outer race. When the relative speed changes to zero, the overrunning clutch transfers from the disengaged state to the engaged state. The other sensor measures the torque direction between the inner race and the outer race. When the torque direction changes, the overrunning clutch transfers from the engaged state to the disengaged state. The script simulation program is used to activate or eliminate the fixed pair between the inner race and outer race.

\section{2) Contact between cam and roller}

During the movement of the device, the main interaction between cam and roller is continuous contact. This kind of contact can be defined by the Restitution model and the Impact function model in ADAMS software. In this simulation, the Impact function model is used to define the contact.

The parameters related to the contact of cam and roller in this simulation are shown in Table III.

TABLE III. THE PARAMETERS OF THE CONTACT

\begin{tabular}{|c|c|}
\hline Parameters & Value \\
\hline Stiffness(N/mm) & $6.74 \times 10^{5}$ \\
\hline Force Exponent & 2.2 \\
\hline Damping(N·s/mm) & 70 \\
\hline Penetration Depth(mm) & 0.01 \\
\hline Static Coefficient & 0.10 \\
\hline Dynamic Coefficient & 0.08 \\
\hline Stiction Transition Velocity(mm/s) & 0.1 \\
\hline Friction Transition Velocity(mm/s) & 1.0 \\
\hline
\end{tabular}

3) Determination of friction moment in the testing device

All the rotating shafts in a machine are supported by the bearing. The part of the shaft in the bearing is called journal. The journal and the bearing constitute the rotating pair.

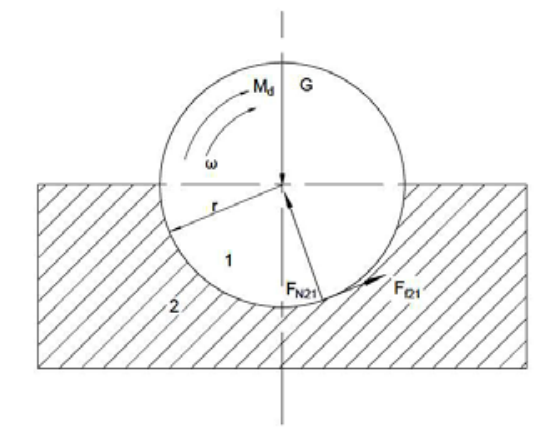

FIGURE III. THE ANALYSIS OF THE JOURNAL

As is shown in Figure III, the journal with a radial load $G$ is rotating in the bearing under the action of the driving force moment $M_{d}$. At this time, the friction moment produced by the two elements will to prevent the journal's rotation. Then the friction of the bearing to the journal can be described as[10]:

$$
F_{f 21}=f_{v} G
$$

where

$$
f_{v}=(1 \sim \pi / 2) f
$$

where $f$ is the friction coefficient in the journal. The friction moment of the bearing to the journal can be described as:

$$
M=F_{f 21} r=f_{v} G r
$$

In order to simplify the simulation model, the friction moments calculated by the above equations are added to flywheel, shaft A and shaft B. Their values are shown in Table IV.

TABLE IV. THE FRICTION MOMENTS OF THE MODEL

\begin{tabular}{|c|c|}
\hline Location & Value(N·mm) \\
\hline Flywheel & 300 \\
\hline Shaft A & 3 \\
\hline Shaft B & 3 \\
\hline
\end{tabular}

C. Simulation Results and Analysis of the Testing Device

Figure IV shows the simulation results of the angular velocity variation of the flywheel with two different moment of inertia during the first three cycles of the device's movement. Figure $\mathrm{V}$ shows the simulation results of the torque variation of the torsion spring $\mathrm{A}$ and the overrunning clutch $\mathrm{A}$ during the first cycle. Figure VI shows the simulation results of the torque variation of the torsion spring $\mathrm{B}$ and the overrunning clutch B during the first cycle. Figure VII shows the simulation results of the torque variation of the torsion spring A and the overrunning clutch A during the third cycle. Figure VIII shows the simulation results of the torque variation of the torsion spring $\mathrm{B}$ and the overrunning clutch $\mathrm{B}$ during the third cycle. Figure IX shows the relationship between stiffness coefficient of the torsion spring and the maximum synchronous torque of the clutch.

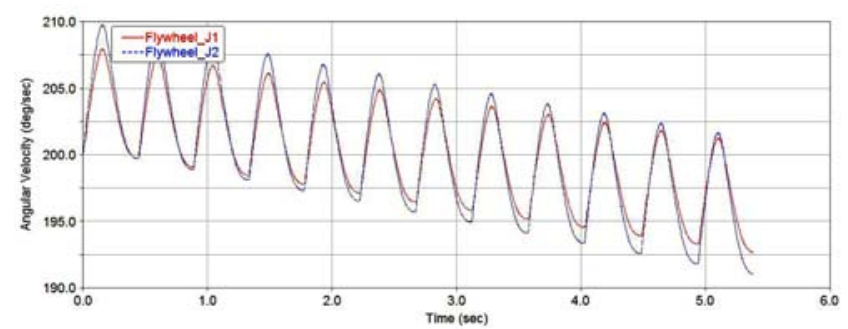

FIGURE IV. THE ANGULAR VELOCITY VARIATION OF THE FLYWHEEL 


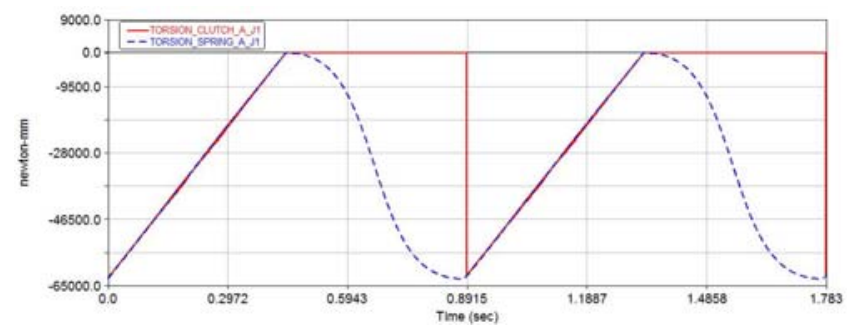

FIGURE V. THE TORQUE VARIATION OF THE TORSION SPRING A AND THE OVERRUNNING CLUTCH A

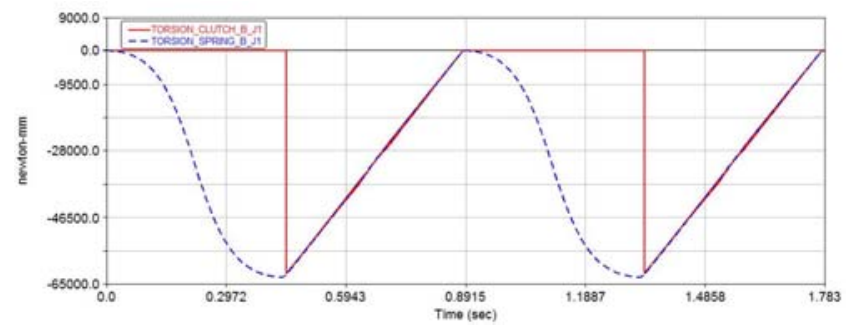

FIGURE VI. THE TORQUE VARIATION OF THE TORSION SPRING B AND THE OVERRUNNING CLUTCH B

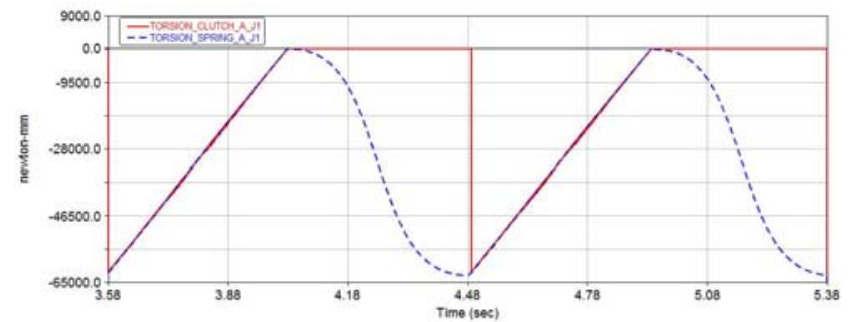

FIGURE VII. THE TORQUE VARIATION OF THE TORSION SPRING A AND THE OVERRUNNING CLUTCH A

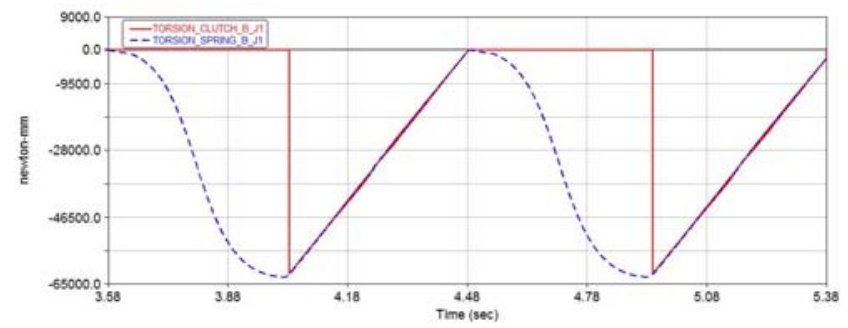

FIGURE VIII. THE TORQUE VARIATION OF THE TORSION SPRING B AND THE OVERRUNNING CLUTCH B

From Figure IV, it can be seen that: During the movement of the device, the rotational speed of the flywheel decreases periodically due to the friction. When the flywheel's moment of inertia is $J_{1}$, its rotational speed decreases by $2.5 \mathrm{deg} / \mathrm{s}$ per cycle, and it decreases the kinetic energy of 6.96J. When the moment of inertia of the flywheel is $J_{2}$, its rotational speed decreases by 3deg/s per cycle, and it decreases the kinetic energy of 6.67J. That means the testing device approximately costs $7 \mathrm{~J}$ energy per cycle. When the initial angular velocity of the flywheel is the same, the greater the flywheel's moment of inertia is, the slower the rotational speed of the device decreases. The flywheel's rotational speed and moment of inertia determine the duration of the experiment. The device can perform multiple cycles, and the motor provides energy to the flywheel when the flywheel rotational speed is decreased to a certain value (such as $100 \mathrm{deg} / \mathrm{s}$ ).

From Figure V to Figure VIII, it can be seen that: The load change of the overrunning clutch $\mathrm{A}$ and $\mathrm{B}$ in the engaged state is basically the same as the torque change of the torsion spring $\mathrm{A}$ and $\mathrm{B}$, respectively. The time of the clutch in the engaged state is the same as that in the disengaged state. Along with the process of the simulation, the cycles of the engaged state and the disengaged state are gradually increasing. The results of the simulation represent that the load of the overrunning clutch satisfies the demands.

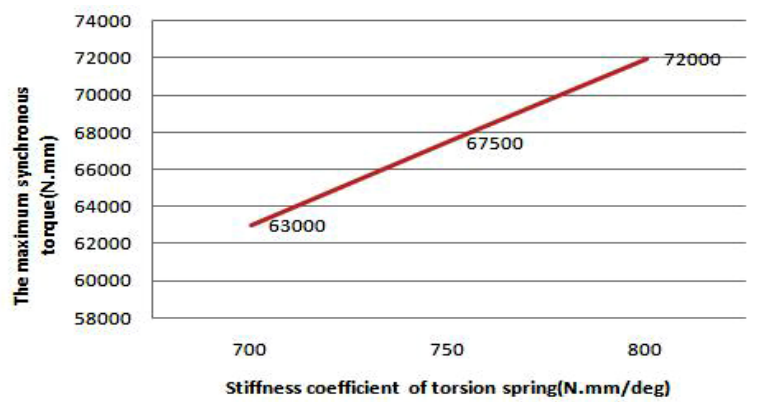

FIGURE IX. THE RELATIONSHIP BETWEEN STIFFNESS COEFFICIENT OF THE TORSION SPRING AND THE MAXIMUM SYNCHRONOUS TORQUE OF THE CLUTCH

From Figure IX, it can be seen that: The maximum synchronous torque of the clutch is proportional to the stiffness coefficient of the torsion spring, and its slope is 90.Therefore, the synchronous torque of the clutch can be controlled by changing the parameters of the torsion spring.

\section{CONCLUSIONS}

This paper proposes an energy-saving overrunning clutch life testing device which structure is simple, layout is symmetrical and energy consumption is low. Following conclusions can be drawn from the study:

The device can achieve the transition of the two working states through its simple mechanical structure. The duration of the clutch's engaged state is the same as the disengaged state. The same function of the traditional overrunning clutch life testing system is achieved by this testing device.

The load, in other words, the synchronous torque of the overrunning clutch in its engaged state can be controlled by the stiffness coefficient of the torsion spring.

The energy loss in the device only includes the friction between the device's components and a small impact between cam and roller. Energy consumption is low.

\section{REFERENCES}

[1] L. Zhu, and G. L. Shi, "The Development of Overrunning Clutch Comprehensive Performance Test Stand," Machinery Design and Manufacture, 2013, 10: 148-151.

[2] X. Y. Tie, Y. X. Hou, Z. C. Zhang, F. Jiang, "Design of Locking Fatigue Tester for Abnormal Roller Overrunning Clutch," Bearing, 2014, 1: 3438. 
[3] S. C. Burgess, T. A. Stolarski, and S. Karp, "Analysis of Failure Modes of a Thermoplastic One-Way Clutch," Proc. Inst. Mech. Eng., Part C: J. Mech. Eng. Sci., 1991, 205: 209-215.

[4] K. Imado, and H. Nakata, "Experimental study of novel clutch utilizing self- locking property of belt," Proc IMechE Part J: J Engineering Tribology, 2013, 227(8): 912-918.

[5] H. Richter, and R. Maynard, "Hybrid-dynamical modelling, characterization, and experimental verification of a free-wheeling clutch," Proceedings of the Institution of Mechanical Engineers, Part I: Journal of Systems and Control Engineering, 2010, 224: 361-372.

[6] C. J. Li, Z. Q. Zhang and X. F. Ji, "Measure and Control System of Aviation Engine Clutch Bear Test bed," Machinery and Electronics, 2004, 6: 32-34.

[7] Y. S. Liu, P. J. Zhang and Z. G. Li, "Test device of overrunning clutch bearing and its control system," Beraning, 2003, 8: 24-26.

[8] P. CRAMTON D, R. J. FERGUSON and J. H. KERR, "An apparatus for dynamic testing of overrunning clutches," Mechanism \& Machine Theory, 1986, 21(5): 375-384.

[9] H. Xu, Mechanical Design Handbook, Beijing, China Machine Press, 2000.

[10] H. Sun, Z. M. Chen and W. J. Ge, Theory of Machines and Mechanisms, Beijing, Higher Education Press, 2006. 